\title{
Las relaciones internacionales y el papel de Brasil en la gobernanza climática global durante el gobierno de Luis Inácio Lula da Silva (2003-2010)
}

International Relations and the Role of Brazil in the Global Climate Governance during the Luis Inacio Lula Da Silva's Government (2003-2010)

Juliane Rodrigues Teixeira Chile juliane.rt@gmail.com

\section{Resumen}

Se destaca la actuación multilateral de Brasil, especialmente durante el gobierno de Luis Inácio Lula da Silva (2003-2010), en medio del debate sobre la temática ambiental presente en las relaciones internacionales contemporáneas y en el contexto de la ampliación de la necesidad de una gestión colectiva de la crisis ambiental debido a su carácter transnacional. Por el papel protagónico del país en las negociaciones multilaterales y por su posición natural privilegiada, en función de su gran biodiversidad, se plantea la perspectiva nacionalista desde principios de los años noventa, cuya representación se ha ampliado en los foros de discusiones globales en la primera década del siglo XXI. Se analiza la gobernanza global del cambio climático. Primero se destacan las principales cumbres mundiales sobre el tema, así como el tradicional posicionamiento de Brasil.

Luego se aborda la actuación brasileña en el ámbito externo, así como las políticas 
de mitigación implementadas durante el gobierno de Lula da Silva. Así, se logra presentar cómo Brasil ha pasado de una postura reactiva hacia una política exterior más activa, participativa y colaborativa, siempre con principios de las responsabilidades comunes, pero diferenciadas, y la preservación de la soberanía sobre sus recursos naturales. La idea de preservar y proteger el medioambiente, se transfiere hacia los países desarrollados y con la visión de financiar y equilibrar las relaciones de poder con los países en desarrollo.

Palabras clave: arte conceptual; fotografía; naturaleza; percepción del tiempo; tiempo fenomenológico; video-instalación.

\begin{abstract}
The article highlights the multilateral action of Brazil, especially during Luis Inacio Lula da Silva's government (2003-2010), in the midst of the debate on environmental issues, present in contemporary international relations, and in the enlargement context of the need of a collective management of the environmental crisis due to its transnational character. Thanks to the country's role in the multilateral negotiations and to its privileged and natural position, in function of its great biodiversity, the article describes the nationalist perspective starting from the beginning of the 1990s, whose representation has been extended to global discussion forums in the first decade of the 21 st century. The global governance of climate change is analysed. First, the major summits on the topic, as well as the traditional positioning of Brazil, are highlighted. Then, the Brazilian action in the external sphere and mitigation policies implemented during the government of Lula da Silva are considered. Thereby, Brazil is described as a country that has moved from a reactive stance to a more active, participatory, and collaborative foreign policy, always with principles of common but differentiated responsibilities, and the preservation of the sovereignty over its natural resources. The idea of preserving and protecting the environment is transferred to the developed countries, with the vision of funding and balancing power relations with developing countries.
\end{abstract}

Keywords: conceptual art nature; perception of time; phenomenological time; photography; video installation.

\title{
Introducción
}

La temática de la gobernanza climática global adquiere cada vez más relevancia en el mundo actual, al tener en cuenta las consecuencias provocadas por la degradación ambiental que llevan a la discusión y a la proliferación de distintos 
acuerdos multilaterales como mecanismos necesarios para contener la destrucción del medioambiente y se ha llega incluso a la discusión sobre la limitación del crecimiento económico como herramienta para garantizar la vida de las generaciones futuras. De este modo, las amenazas globales deben ser enfrentadas en el marco también de la responsabilidad global, para lo que se requieren los esfuerzos de todas las naciones para contener problemas como el cambio climático.

Así, este representa uno de los principales desafíos para los entes tomadores de decisiones y la comunidad internacional en principios del siglo XXI. En este sentido, América Latina y, por consiguiente, Brasil, presentan funciones importantes en la composición de un acuerdo vinculante a nivel internacional, tornando efectiva las medidas de mitigación del problema que puede afectar a todas las naciones debido al incremento de la temperatura del planeta, ocasionado por las emisiones de gases efecto invernadero (GEE), producidas por las actividades derivadas de la quemada de combustibles fósiles y la destrucción de los bosques que actúan como consumidores del dióxido de carbono. El cambio climático es parte de la crisis ambiental global, fenómeno que fue provocado por las prácticas de producción y modelos de consumo impuestos por los países altamente industrializados del llamado Primer Mundo o Norte ${ }^{1}$ (Estenssoro, 2014).

En el caso de mantención de la tendencia actual de emisiones, se prevé su crecimiento exponencial hasta el año de 2030. Por consiguiente, uno de los mayores desafíos de la actualidad es, justamente, eso: buscar el equilibrio entre el crecimiento económico, la reducción de la pobreza, la igualdad social y la protección a los ecosistemas globales que garantizan la manutención de la vida. Para lograrlo,

1. Se entiende por crisis ambiental global una serie de problemas de carácter ecológico y ambientales de alcance mundial generados por el modelo de desarrollo y crecimiento económico de las sociedades altamente industrializadas del Primer Mundo o Norte y que, genéricamente, se sintetiza como la "Civilización Industrial" (civilización que comenzó a desarrollarse con el inicio de la Revolución Industrial alrededor de 1750). Si bien estas sociedades altamente industrializadas alcanzaron un elevado nivel de desarrollo y estándar de vida para su población, generaron problemas de tan enorme magnitud que pusieron en riesgo, por primera vez en la historia, la continuidad de la vida del ser humano en el planeta, así como la propia continuidad de la vida en él. En un primer momento, las macro variables que componían esta crisis ambiental se referían a fenómenos tales como: la contaminación, la pérdida de la biodiversidad, el cambio climático, el agotamiento de los recursos naturales, la destrucción de la capa de ozono, y la llamada, por algunas partes, "explosión demográfica". Posteriormente, y tras múltiples debates en el seno del sistema internacional, caracterizada por una clara confrontación política y teórica entre el Norte y el Sur se incorporó también, como variable generadora de esta crisis ambiental, el tema de la pobreza y miseria en que vive gran parte de la humanidad (Estenssoro, 2014). 
es fundamental la cooperación de todas las naciones en la elaboración e implementación de acuerdos ambientales eficientes que contengan medidas efectivas de mitigación y conservación de los recursos naturales, que fortalezcan los mecanismos de gobernanza ambiental global y que tomen en cuenta la globalización y la extensión de las amenazas ambientales que no respetan fronteras.

Por ende, para lograr la reducción de las emisiones de GEE, es necesario establecer políticas, inversiones y financiamientos públicos y privados, transferencia de tecnología a nivel internacional y acciones concretas en el ámbito local. Estos hechos necesitan acuerdos internacionales y políticas nacionales en los sectores más extensivos en el uso de combustibles fósiles, especialmente en la generación de energía eléctrica, industria, transporte y construcción. Igualmente, no solo se necesita la cooperación de los países desarrollados, sino también la participación de las grandes economías emergentes y en vías de desarrollo, como China, India, Brasil, Rusia, entre otros, que representarán la mayor parte de la proyección de emisiones en las décadas siguientes (Quirola, 2009).

También es importante señalar la participación de Brasil en los debates internacionales, ya que el país ocupa una posición natural privilegiada, en función de su gran biodiversidad. Así ha participado activamente, ejerciendo incluso el papel de liderazgo en algunos acuerdos internacionales que tratan del cambio climático, al ser el primer país en firmar la Convención de las Naciones Unidas de 1992, al igual que uno de los idealizadores del Mecanismo de Desarrollo Limpio implementado en Kyoto y protagonista al comprometerse voluntariamente en la reducción de $36 \%$ hasta $39 \%$ de las emisiones GEE hasta el 2020, en la CPO15 en Copenhague. Además, ha participado activamente en las negociaciones sobre la extensión del Protocolo de Kioto, en 2010, en Cancún (Brito, 2011).

Por ende, durante el gobierno de Luis Inácio Lula da Silva, una serie de avances pudieron ser observados, especialmente en cuanto a las cuestiones sociales a través de implementación de nuevas políticas públicas de transferencia de renta. La esfera ambiental, por su parte, presentó una dualidad en relación con la debilidad en las políticas internas y un mayor protagonismo de la diplomacia brasileña en los foros multilaterales. Así, el medioambiente enfrentó a nivel interno el mismo proceso contradictorio presente en la política internacional sobre cómo armonizar el desarrollo y el crecimiento económico juntamente con la preservación ambiental. De este modo, a pesar de una serie de políticas públicas aplicadas a las cuestiones socioambientales, conviene destacar los avances, incluso los retrocesos y 
los diversos puntos de conflicto que marcaron la agenda de su gobierno (Kageyama y Santos, 2012).

Así, pese la insuficiencia de algunas políticas en el ámbito interno, fue en el campo de las relaciones internacionales donde la diplomacia del gobierno Lula da Silva logró ampliar la participación brasileña en la elaboración de las reglas en los regímenes de gobernanza global, especialmente a través del incremento de la cooperación Sur-Sur en los distintos foros de diálogo, donde se destaca la cooperación con naciones emergentes como India y Sudáfrica, países en desarrollo que enfrentan dilemas ambientales semejantes (Visentini y Silva, 2010). Entre sus reivindicaciones, están la ampliación de la participación de los países en vías de desarrollo en la elaboración de las reglas sobre la mitigación de los daños ambientales, especialmente en el régimen internacional del cambio climático. En este sentido, la diplomacia de Lula da Silva enfatizó su posición de que los países desarrollados poseen una mayor responsabilidad (Barros-Platiau, 2010).

En las líneas siguientes, primero se analiza la gobernanza global del cambio climático, donde se desatacan las principales cumbres mundiales sobre el tema, así como el tradicional posicionamiento de Brasil. Posteriormente, se aborda la actuación brasileña en el ámbito externo, así como las políticas de mitigación implementadas durante el gobierno de Lula da Silva, comprendidas en el período del 2003 hasta el 2010.

\section{La gobernanza global del cambio climático}

\section{- La gobernanza global}

El sistema internacional contemporáneo se caracteriza por la dinámica de intereses que evidencian distintos modelos de desarrollo económico presentes en las naciones, centrados en la división entre los países que buscan mantener las tradicionales formas de desarrollo a partir del uso de combustibles fósiles, con alto impacto en la emisiones de GEE, principales causadores del cambio climático, y la emergencia de países reformistas, que intentan reformular el modelo de desarrollo hacia la transición a una economía de bajo consumo de carbono. Estados Unidos, Canadá, China, India y Rusia son ejemplos del primer grupo, que resisten a la elaboración de un nuevo acuerdo global más ambicioso; mientras que la Unión Europea, Japón, Sudáfrica y Corea del Sur son ejemplos del segundo grupo de países (Viola, 2010). Así, el cambio depende de un acuerdo internacional 
que sea capaz de: Estimular las inversiones en recursos y medidas de mitigación que generen eficiencia energética.

a. Expandir la utilización de energías renovables para reemplazar las tradicionales fuentes de combustibles fósiles por la energía solar y eólica.

b. Utilizar biocombustibles que no influencien en la producción de alimentos y que tampoco generen más deforestación.

Igualmente, la ciencia y la tecnología son herramientas esenciales en la transición hacia una economía baja en carbono (Quirola, 2009).

Por ende, la gobernanza ambiental global es imprescindible. Ella comprende a todos los actores, instrumentos políticos, procedimientos, normas y mecanismos financieros que regulan la protección del medioambiente en todo el globo, junto las iniciativas y mecanismos, gubernamentales o no, propuestos para lograr objetivos comunes. De este modo, comprende las líneas generales que orientan la conducta de los estados, sociedad civil y del mercado en busca de objetivos a largo plazo; engloba igualmente la emergencia de nuevos actores y mecanismos políticos globales que pueden llevar a la limitación de la soberanía de los estados frente a la cada vez mayor interdependencia global y requiere de la cooperación multilateral.

No obstante, es importante señalar que el régimen internacional del medioambiente se comparado con otros regímenes como el de comercio, por ejemplo: fragmentado y carente de articulación para tornarlo más eficaz en el cumplimiento de los objetivos ambientales a nivel global, pues refleja dispersión, superposición de otros intereses y falta de articulación en las políticas ambientales; se resaltan las dificultades en responder adecuadamente a los desafíos ambientales.

Entre los pilares que conforman la gobernanza global del cambio climático está la cooperación a largo plazo como instrumento para lograr, de forma eficaz, la reducción de las emisiones; la necesidad de medidas especiales para los países en desarrollo, a partir de la transferencia de tecnologías, que apoyen las medidas de mitigación y adaptación a los problemas causados por el cambio climático; la consolidación del mecanismo financiero, que establezca recursos e inversiones capaces de estimular las medidas de mitigación y adaptación, a través de acuerdos concretos en estos ámbitos, especialmente en la transferencia de tecnología y adaptación que amplían las capacidades de desarrollo de estas naciones (Quirola, 2009). 
Igualmente, lleva en cuenta la responsabilidad compartida entre todas las naciones, pero diferenciada, puesto que la acumulación de las emisiones GEE es de responsabilidad primordial de los países desarrollados, razón por la cual se les impone la responsabilidad de asumir compromisos en materia de financiamiento y transferencia de tecnologías para los países en vías de desarrollo. De este modo, debe ser respetado el derecho al desarrollo de las naciones que enfrentan grandes problemas de pobreza y desigualdad, mediante la búsqueda y establecimiento de políticas que minimicen los efectos negativos en la transición hacia una sociedad baja en el consumo de carbono. No obstante, los intereses económicos tornan las posiciones divergentes entre las naciones, especialmente en la promoción de un acuerdo vinculante de reducción de emisiones para los países desarrollados, pues se encuentran obstáculos en cuanto a las metas de reducción entre los propios países industrializados, en la ampliación de sus inversiones en mitigación y en sus compromisos para financiar la adaptación al cambio climático (Quirola, 2009).

América Latina, por su parte, pese la existencia de actividades industriales, transportes, agricultura y producción de residuos que emiten GEE, concentra en la deforestación, degradación y cambio del uso del suelo sus principales fuentes de emisiones, lo que ocasiona pérdidas de biodiversidad e impactos sociales negativos para las comunidades a partir de los cambios generados por el calentamiento global y afecta especialmente a las poblaciones más pobres (Quirola, 2009).

Ya Brasil es considerado una potencia media en el ámbito de la lucha en contra del cambio climático, es responsable por cerca de $5 \%$ de las emisiones, pertenece al conjunto de países cuyo acuerdo es fundamental para la reducción de los problemas generados por el cambio climático. Sus políticas pueden ser consideradas como reformistas desde el punto de vista de los objetivos de reducción de emisiones, pero también conservadoras, en la medida en que ha mantenido una fuerte alianza con países como China -el mayor emisor-e India, que detienen posiciones resistentes a un gran acuerdo mundial efectivo, juntamente con Estados Unidos, en contraste con la postura reformista expresada sobre todo por la Unión Europea (Viola, 2010).

\section{- El histórico del debate sobre el cambio climático}

La temática ambiental fue introducida en la agenda de la política mundial a partir de 1972, con la realización de la Conferencia de Estocolmo, momento en que la 
preocupación con el medioambiente era considerada una condicionante al tradicional modelo de desarrollo y crecimiento económico; fue necesario, posteriormente, establecer el concepto de desarrollo sustentable para conciliar el crecimiento económico juntamente con el desarrollo humano y ambiental. En 1988 se creó el IPCC, el Panel Intergubernamental sobre el Cambio Climático, se intensificaron las investigaciones sobre el cambio climático, especialmente a partir de la influencia de los factores antropogénicos que interferían en el aumento de la temperatura del planeta (Reggiani, 2008).

Posteriormente, se ha llevado a cabo conferencia de las Naciones Unidas para debatir las estrategias de desarrollo sustentable Rio 92, donde se instituyó la Convención Marco del Cambio Climático Global. Aquí se señaló que la intervención humana en el medioambiente estaría afectando de modo directo el clima del planeta a partir del incremento de las emisiones de GEE.

Por consiguiente, a partir de este momento se realizaron una serie de convenciones para reglamentar las emisiones de los gases, denominadas Convenciones de las Partes. La COP1 fue la primera en efectuarse en Berlín, en 1995, la cual marcó el inicio efectivo de las negociaciones sobre el tema al proponer el establecimiento de un protocolo que regule las emisiones de GEE (Reggiani, 2008).

La COP3, realizada en Kioto en 1997, dio lugar a la creación del Protocolo de Kioto para reglamentar las emisiones. Se establecieron objetivos de reducción a los países de acuerdo con su nivel de desarrollo, los cuales se dividieron entre países del Anexo I, los países desarrollados y los demás países. Con el fin de promover la transparencia en la información prestada por los estados, y para intensificar la cooperación entre ellos, el acuerdo que entró en vigor en 2005 buscó incentivar la reducción de las emisiones considerando el principio de la responsabilidad común, pero diferenciado entre las naciones, de acuerdo con su grado de desarrollo. Así, los países integrantes del Anexo I tendrían objetivos de reducción de emisiones vinculantes, mientras que los demás países, los en vías de desarrollo, no tendrían metas concretas de reducción ${ }^{2}$. Para los países que no pertenecen

2. Para motivar la cooperación entre los países y reducir las emisiones de GEE fueron implementados tres mecanismos de flexibilización, en Mecanismo de Implementación Conjunta y el de Emisiones Comerciables, permitidos solamente entre los países del Anexo I, se estableció la posibilidad de implementar medidas de reducción en conjunto y comercializar el exceso de la reducción generada; y el Mecanismo de Desarrollo Limpio, que contó con la participación activa de Brasil en las negociaciones, promoviendo el desarrollo limpio en los países en vías de desarrollo a través de las inversiones de países desarrollados (Reggiani, 2008). 
al Anexo 1, entre ellos Brasil, el protocolo no prevé metas de reducción efectiva, puesto que su proceso de desarrollo está asociado al predominio de energías esencialmente contaminantes. No obstante, el acuerdo apunta la necesidad de todos los países en disminuir las emisiones de GEE (Reggiani, 2008).

\section{- La tradicional posición brasileña en el debate sobre el cambio climático}

La posición de Brasil sobre la cuestión climática ha variado durante el período de 1972 hasta 1990. Esto, debido a que, durante la I Conferencia de las Naciones Unidas sobre el medioambiente, realizada en Estocolmo, el país creía que el principal problema de los países en vías de desarrollo era la pobreza y consideraba la protección ambiental un aspecto secundario en el proceso de desarrollo económico. De este modo, juntamente con China, Brasil ejerció el liderazgo junto a los países periféricos: defendían la soberanía nacional en cuanto al uso de los recursos naturales; la protección ambiental, solamente después de lograr mayor crecimiento económico y mejores niveles de vida para la mayor la parte de la población; y la responsabilidad de la protección ambiental global debería ser asumida exclusivamente por los países desarrollados (Quirola, 2009).

Por lo tanto, para Brasil, los países desarrollados poseían una mayor responsabilidad, en vista de que, desde la Primera Revolución Industrial (siglo XIX) emiten GEE, con lo que representan una amenaza para toda la humanidad. Por lo tanto, la crisis ambiental tiene sus bases en el desarrollo económico de los países del Norte, centrados en un sistema económico depredador y contaminante (Estenssoro, 2010). No obstante, el aumento de la conciencia ambiental global llevó a la menor aceptación de los procesos productivos excesivamente contaminantes, lo que posibilitó que el país buscase adaptarse a las nuevas necesidades del mercado a través de la transformación hacia procesos productivos menos contaminantes y la implementación de la Política Nacional del Medioambiente, a partir de 1981.

Además, el cambio en el posicionamiento de Brasil fue facilitado también por la crisis del modelo económico basado en la explotación masiva de los recursos naturales, precedido por la mayor participación activa del país, junto a los foros internacionales, lo que llevó a su adhesión a los regímenes impulsados por los países desarrollados. Esta fue una forma de atribuir mayor credibilidad al país a principios de la década de 1990, momento en que la nación abría su economía y adhería a la agenda neoliberal. Así, pronto el país organizaba la Rio 92 como forma de demostrar esta nueva responsabilidad internacional (Quirola, 2009). 
Por consiguiente, los cambios en la visión del país sobre los problemas ambientales también alteraron su tradicional posicionamiento internacional, actuando activamente en la elaboración de la Agenda 21 sobre el desarrollo sustentable. Para Brasil, los problemas ambientales pasaron a ser de extrema importancia y fue necesaria la concientización de toda la comunidad internacional; no obstante, la responsabilidad de estos problemas y las soluciones adoptadas deberían ser diferenciadas en cuanto al grado de desarrollo de las naciones. Igualmente, fue importante su participación en la realización de la COP 1 en Berlín, en 1995, al plantear que los países en desarrollo no deberían ser obligados a implementar metas de reducción de emisiones, posición mantenida en las reuniones siguientes, con el argumento de que la introducción de obligaciones de reducción en las emisiones de GEE podría afectar al modelo de desarrollo y el crecimiento económico de los países más pobres (Quirola, 2009).

Además, merece destacarse la participación brasileña en las negociaciones del Protocolo de Kioto, el acuerdo multilateral más importante firmado hasta el momento para contener el avance de las emisiones, como resultado de la COP 3, realizada en Kioto, en 1997. Brasil es signatario del acuerdo y, pese a no estar sometido a objetivos cualitativos de reducción de emisiones conforme los países desarrollados, debe tomar medidas que conlleven a la disminución de estas (Brito, 2011).

Igualmente, las negociaciones del protocolo se extendieron desde 1996 hasta 2001, momento en que el país actuó activamente pautado por sus principales intereses, como por ejemplo: defensa del desarrollo como herramienta fundamental del orden mundial; la promoción del desarrollo asociado a la sustentabilidad ambiental, teniendo en cuenta el crecimiento del tema ambiental en el país a través de las políticas implementadas; la promoción del mayor liderazgo brasileño en el mundo, debido al aumento del prestigio del país logrado durante el gobierno de Fernando Henrique Cardoso, al adherir a la agenda elaborada por los países desarrollados; y búsqueda por evitar la reglamentación internacional del uso de los bosques, que obstaculiza que otros países violasen la soberanía brasileña sobre el territorio amazónico al cuestionar el uso económico que el país atribuía a este ecosistema. Así, el país se ha posicionado de modo activo en las negociaciones, buscando resguardar los intereses de los países en vías de desarrollo (Reggiani, 2008). 


\section{Brasil y la gobernanza global del cambio climático durante el gobierno de Lula da Silva}

Desde la década de 1990 hasta 2005, Brasil adoptó una postura de carácter más conservador en la gobernanza global del clima, alterando con períodos en que ejerció un mayor liderazgo reformista. No obstante, con la consolidación de políticas de control de la deforestación a partir de 2006, el país pasó a adoptar posiciones más reformistas, que culminaron con la adopción del compromiso de reducción voluntaria de emisiones asumido en la COP15, en 2009 (Viola y Franchini, 2013).

Por ende, para comprender la participación de Brasil en el Régimen del Cambio Climático, además de analizar su política interna, también es necesario considerar los orígenes de sus emisiones, puesto que ayudan a guiar su posicionamiento, ya que el país es un gran emisor de GEE causados por la deforestación y mal uso del suelo, puesto que posee una matriz energética considerada limpia. Esto es importante, en la medida que el Informe de Evaluación del IPCC de 2007 establece que $57 \%$ de las emisiones mundiales son provenientes de energía fósil y $18 \%$ por la deforestación y mal uso de la tierra (Reggiani, 2008). De este modo, la transición del posicionamiento brasileño hacia planteamientos más reformistas en el ámbito del cambio climático está asociado con sus dinámicas de emisiones, las que, a partir de los años 2000, pasaron por profundos procesos de cambios en su trayectoria, desde la adopción de políticas internas que lograron reducir las provenientes de la deforestación en la Amazonia y, consecuentemente, las emisiones originadas por este sector (Viola y Franchini, 2013).

\section{- La participación en la COP12}

Desde la COP12, realizada en Nairobi en el año de 2006, la ministra de Medioambiente, Marina Silva, ya defendía los cambios en la matriz energética, que requieren transformaciones también en el modelo económico centrado en estándares ya no más aceptables de producción y consumo. Además, teniendo en cuenta las características singulares de las emisiones brasileñas, sostenía la posición de reforzar el control de la deforestación, especialmente en la Amazonia, a partir de cambios fundamentales en el modelo de desarrollo del país, atacando las causas y los vectores económicos involucrados en el problema, así como estableciendo nuevos mecanismos de desarrollo sustentable. Por ende, el país abandonaba la tradicional posición de evitar que el uso de los bosques fuera objeto de 
reglamentación internacional, proponiendo, en esta oportunidad, la creación de un fondo para evitar la deforestación. De este modo, Brasil se mostraba dispuesto a aceptar la reglamentación internacional (Reggiani, 2008).

\section{- La participación en la COP13}

El gobierno de Lula da Silva defendió la mitigación del cambio climático persistiendo en la necesidad de creación de mecanismos de adaptación principalmente en los países menos desarrollados a partir de la mayor cooperación y transferencia de tecnología. Esta postura fue reiterada durante la realización de la COP13, en Bali, Indonesia, en el año 2007, cambiando la tradicional posición del país en principios de la negociación del Protocolo de Kioto, en que los países en desarrollo no deberían pagar por la mitigación del cambio climático, pasando a destacar la necesidad de creación de mecanismos de adaptación, financiamiento y transferencia de tecnología a los países en desarrollo, para que estos puedan ayudar en la mitigación del cambio climático. De este modo, Brasil pasaba a destacarse como uno de los líderes del G77 + China, agrupación que reúne a los países en desarrollo (Reggiani, 2008).

Igualmente, en esta oportunidad, la actuación del país resaltó la necesidad de implementar medidas de reducción de las emisiones provenientes de la degradación de los ecosistemas forestales en todo el mundo, ya que el país consideraba bajos los incentivos para la cooperación en los países en desarrollo para la reducción de las emisiones provenientes de la deforestación. Así, señalaba la necesidad de incrementar las transferencias financieras y tecnológicas para auxiliar a los países en desarrollo que tomaran estas medidas (Reggiani, 2008).

\section{- La destacada participación en la COP15}

La COP15 fue un acuerdo conducido por un grupo limitado de países, en donde destacó la participación de Brasil en el Acuerdo de Copenhague. Entre sus principales objetivos estaban el de negociar, redactar y aprobar nuevas metas de reducción de las emisiones relativas a la segunda parte del Protocolo de Kioto, a ser cumplidas a partir de 2013. No obstante, no fue posible llegar al consenso necesario para la adopción del documento, pero Brasil propuso que los países en desarrollo también deberían reducir sus emisiones al establecer voluntariamente un plan para la reducción entre $36 \%$ y $39 \%$ de sus emisiones hasta 2020 , cambiando la tradicional postura del país en rechazar objetivos a los países en desarrollo (Brito, 2011). 
No obstante, es importante señalar que el hecho del país haber asumido el compromiso de reducción de forma voluntaria no implica que haya abandonado la división entro los países en desarrollados y en vías de desarrollo, es decir, entre los pertenecientes al Anexo I del Protocolo de Kioto, y los no pertenecientes. Para Brasil, los compromisos voluntarios para los países emergentes no deberían tornarse objetos de reglamentación internacional, sino una expresión de la voluntad de cada país en disminuir sus propias emisiones (Viola y Franchini, 2013). Lo que sí deberían considerar las propuestas es la responsabilidad histórica; la equidad; las capacidades de cada país; así como el reconocimiento del derecho de los países en desarrollo a lograr el crecimiento económico sostenido, erradicando la pobreza. De este modo, las responsabilidades deberían ser comunes, pero diferenciadas, de acuerdo con el grado de desarrollo de las naciones (Quirola, 2009).

Por ende, en el ámbito internacional, se puede observar la existencia de posiciones que convergen en cuanto a la responsabilidad compartida, pero diferenciada para combatir el cambio climático ${ }^{3}$. No obstante, no hay consenso respecto a las metas de reducción, tampoco a los mecanismos de financiamientos en función de los altos costos, pues permanecen posiciones divergentes en los países industrializados y en economías en desarrollo, como China, que defiende primeramente su derecho al crecimiento económico (Quirola, 2009).

En las negociaciones previas a la COP15, en la Conferencia sobre el Cambio Climático realizada en 2009, en Copenhague, hubo pocos avances; solamente la Unión Europea y Japón, los únicos entre los actores más relevantes, se

3. Pero para otros, como la Unión Europea, por ejemplo, el cambio climático debe ser enfrentado por toda la comunidad internacional, sean los países desarrollados o en vías de desarrollo, buscan alcanzar $30 \%$ de las emisiones globales hasta 2020, en comparación con los niveles de 1990, y $50 \%$ hasta 2050. Ya para la mayoría de los países petroleros, los acuerdos no pueden afectar el crecimiento de los países en desarrollo. Para Japón, debe existir un único objetivo en el plazo de reducción de emisiones; además, todos los países deben implementar acciones de mitigación de forma solidaria en el marco de las responsabilidades comunes pero diferenciadas, así, la responsabilidad por contener el avance del cambio climático debe ser de todos los países. Ya para China, los países desarrollados deben recortar sus emisiones para mantener el estado atmosférico adecuado que posibilite el desarrollo sostenible y la erradicación de la pobreza por parte de los países en vías de desarrollo, así como la reducción de $40 \%$ de las emisiones hasta 2020, con base en 1990. América Latina, por su parte, negocia de forma individual o en bloques subregionales, ya que hay divergencias en cuanto a los mecanismos que deben ser incluidos en los compromisos como la COP15. Ya Brasil actúa a partir de un liderazgo individual, centrado en su territorio amazónico. Así, se torna cada vez más necesario establecer acuerdos políticos regionales que tomen en cuenta el cambio climático como tema prioritario (Quirola, 2009). 
comprometieron con objetivos significativos de reducción de las emisiones de GEE con base en los números de 1990. No obstante, Brasil cambió su posición e ingresó al grupo de los grandes emisores para proponer, voluntariamente, metas significativas para la reducción de $10 \%$ en relación con 2008. Además, Francia y Brasil habían anunciado una asociación estratégica en busca de un acuerdo relevante para contener el cambio climático en Copenhague, y critican las posiciones conservadoras de distintos países, incluso de Estados Unidas y China, evidenciando el distanciamiento de la alianza con el este último país e India, al aproximarse a la Unión Europea en lo que dice respecto a la agenda del cambio climático (Viola, 2010).

Sin embargo, en este mismo período, las naciones pertenecientes a la APEC Asociación de Países de Asia-Pacífico- lideradas por Estados Unidos y China, consideraron irrealista el proyecto, y abandonaron el compromiso de firmar un acuerdo vinculante sobre la reducción de emisiones de GEE en Copenhague. Dinamarca se distanciaba de la posición de la Unión Europea, y se adhiere a la posición conservadora de Estados Unidos, China e India, lo que caracteriza un retroceso en las metas de reducción propuestas por los grandes países emisores, mientras que Brasil se aproximaba, cada vez más, a los objetivos reformistas planteados por la Unión Europea (Viola, 2010).

Pese a este escenario, Estados Unidos anunció un plan de reducción de $4 \%$ de las emisiones hasta 2020, con base en 1990, aunque requiera la aprobación del Congreso Nacional de este país. China, por su turno, se comprometió a reducir la intensidad de carbono en la conformación de su PIB, de $40 \%$ a $45 \%$ entre 2005 y 2020, sin, establecer un nivel máximo de emisiones. Lo mismo sucede en relación con India y Rusia, que anunciaron políticas para mejorar la eficiencia energética, pero también se negaron a asumir cualquier acuerdo internacional vinculante (Viola, 2010). Además, hay que destacar que la emergencia de la crisis financiera de 2008, que afectó principalmente a los países desarrollados, impactó negativamente las negociones, obstaculizando los acuerdos sobre las perspectivas de mitigaciones del cambio climático que perdieron espacio para las preocupaciones económicas internacionales (Viola, 2010).

Ante todas las reticencias, en vísperas del inicio de la COP15, representantes de las potencias emergentes, Brasil, China, India y Sudáfrica, juntamente con Sudán, el país que entonces detenía la presidencia de la agrupación G77, que congrega a los países en vías de desarrollo, se reunieron para definir una posición en 
común. En este momento, estas naciones rechazaron el establecimiento de metas de reducción de emisiones de carácter obligatorio; rechazaron también sujetar sus políticas sobre el clima a la injerencia internacional en caso en que no hubiera el financiamiento de los países desarrollados; repudiaron la imposición de estándares máximos para el crecimiento de sus emisiones; así como la cobranza de tasas sobre sus exportaciones aplicadas en función del cambio climático, como medidas de antidumping. De este modo, se evidenciaban las distintas visiones de Brasil y Sudáfrica, por un lado, y China, India, Sudán, entre otros, por otro. Esto llevó a la preponderancia de las políticas conservadoras y disminuyó el efecto de la asociación estratégica entre Brasil y Francia en la lucha contra el cambio climático a nivel global (Viola, 2010).

Por ende, las negociaciones finales de la COP15 fracasaron, demostrando las dificultades en lograr un acuerdo vinculante a nivel mundial para mitigar el avance y los efectos del cambio climático. Esto, debido a la oposición de la mayoría de los países emergentes y en vías de desarrollo, además de que las tres grandes potencias climáticas, es decir, las que más contribuyen al cambio climático, Estados Unidos, China y Unión Europea, solamente los últimos eran favorables a establecer un acuerdo con objetivos más relevantes para la mitigación de los problemas. China, por su turno, se mostró como el país más conservador en las negociaciones, mientras que Brasil presentó una posición de establecer metas de reducción voluntaria consideradas reformistas (Viola, 2010).

Por consiguiente, se hace cada vez más importante la participación brasileña en las negociaciones acerca del cambio climático. En este sentido, merece destacarse la propuesta de Lula da Silva para la reducción de las emisiones de GEE en Copenhague, en 2009. Aunque la Cumbre no alcanzó un consenso sobre la responsabilidad y la efectiva reducción de las emisiones entre los países desarrollados y en vías de desarrollo, la propuesta brasileña de reducir voluntariamente sus emisiones estimuló a los países emergentes a hacerlo. Asimismo, hay que destacar que la alternativa alcanzada en la Cumbre de Copenhague fue un acuerdo que, a pesar de contar con la participación de diversas naciones durante las negociaciones, las principales decisiones fueron tomadas por Estados Unidos, Brasil, India, China y Sudáfrica, evidenciando la ampliación de la participación de las principales economías emergentes en la gobernanza global (Amorim, 2010, p. 220).

En 2010, países como Estados Unidos, Unión Europea, Canadá, Japón, México y Corea del Sur, Brasil, Sudáfrica y, posteriormente, China, India y Rusia se 
asociaban al Acuerdo de Copenhague, transformándolo en el acuerdo político internacional más representativo sobre el clima desde la entrada en vigor de la Convención del Cambio Climático en 1994, y llegó a alcanzar a 110 países y 80 $\%$ de las emisiones globales. No obstante, a diferencia del Protocolo de Kioto, no presenta valor legal, es decir, no es vinculante, por lo tanto, no necesita ser ratificado por los parlamentos, dependiendo solamente de la voluntad de cada país en cumplir las metas establecidas (Viola, 2010).

\section{- La participación en la COP16}

La COP16, realizada en Cancún, en el año de 2010, culminó con la extensión del Protocolo de Kioto para más allá del año 2012 y la creación del Fondo Verde para administrar las donaciones de los países ricos, en busca de ayudar a los países en desarrollo a enfrentar los problemas del cambio climático. De hecho, Brasil, juntamente con Reino Unido, obtuvieron un papel político de destaque en la reunión, al llevar a cabo las negociaciones sobre la extensión del Protocolo de Kioto, ya que países como Japón no aceptaban la manutención de este caso; Estados Unidos y China, los grandes emisores, no se adhirieron al acuerdo. Por otro lado, Brasil, China e India se comprometieron a divulgar informes periódicos sobre las emisiones y las medidas a ser implementadas para reducirlas (Brito, 2011).

Por fin, para comprender la participación de Brasil en el Régimen del Cambio Climático, es necesario conocer las dinámicas de la política interna que permiten o no la interiorización de las normas discutidas en el ámbito de la política exterior, ya que el proceso de negociación que empieza en el nivel internacional debe pasar al nivel interno, momento en el que se decide si los acuerdos serán o no implementados en el ordenamiento jurídico nacional.

\section{La dimensión interna de la política climática de Lula da Silva}

Fue durante el primer mandato de Luis Inácio Lula da Silva (2003-2006) que se construyeron las bases de toda su gestión, estableciendo las prioridades en la agenda ambiental de su gobierno. Sus medidas se centraban en la promoción del desarrollo con características más sustentables, tanto en el aspecto ambiental, así como en el económico y social; igualmente, promovió una mayor participación de los sectores sociales en los procesos decisorios, a través de una mayor descentralización de la participación en las decisiones a nivel local; fortaleció el Sistema Nacional de Medioambiente, compartiendo la gestión ambiental con los demás entes de la nación, como previsto por la Constitución brasileña; y a partir de una 
visión transversal ${ }^{4}$ del problema, buscó introducir la agenda ambiental en las demás agendas de otros ministerios, por medio de una actuación pluralista, que influyó en los distintos sectores del gobierno y de la sociedad, aunque no obtuviera grandes resultados en virtud de la preponderancia del interés económico sobre los demás (Kageyama y Santos, 2012).

Por ende, se esperaba, con gran expectativa, un cambio en la política interna brasileña a partir de la introducción de nuevos parámetros de desarrollo. No obstante, sería necesario más que solamente la voluntad política para cambiar los rumbos del país, debiéndose también comprender la complejidad del proceso político dentro de una nación, en virtud de la existencia de intereses distintos entre los diversos actores políticos y sociales, e incluso dentro de la propia coalición que formaba el gobierno. Así, este período fue marcado por una serie de contrariedades en el campo ambiental como forma de Lula da Silva para mantener su apoyo político y su capacidad de gobernabilidad (Kageyama y Santos, 2012).

Por ende, hasta finales de su gobierno, la implementación de políticas efectivas de mitigación y adaptación al cambio climático habían sido limitadas, si se tiene en cuenta que los presupuestos de los Ministerio del Medioambiente y del Ministerio de Ciencia y Tecnología, responsables de dar a conocer el Informe nacional de emisiones, herramienta fundamental para la definición de la política climática nacional e implementación de los cambios tecnológicos en la transformación hacia una economía baja en carbono, eran limitados. Asimismo, hay que señalar que en 2007 fue creada la Secretaría sobre el Cambio Climático, y el año posterior, el mandatario envió el Proyecto de Ley del Cambio Climático al Congreso, pese los problemas en la internalización del cambio climático en la estructura jurídica del país (Viola, 2010).

\section{- La deforestación como principal vector de las emisiones}

En 2008 fue anunciado el Plan Nacional de Cambio Climático, lo que significó la ruptura del tradicional discurso brasileño en relación con la Amazonia, pasando a proponer mayores metas de reducción de la deforestación en la región. Esto es importante en la medida en que, a pesar de su mayor crecimiento económico e

4. La agenda ambiental adquiere cada vez más un carácter transversal, sea a nivel interno o incluso a nivel internacional, influyendo en diversas otras agendas y organismos, ultrapasando, así, a los sectores técnicos y restrictos a su campo (Kageyama y Santos, 2012). 
industrial en los últimos años, el perfil de emisiones brasileñas siguió siendo singu$1 a^{5}$, ya que, hasta esta época, la mitad de las emisiones provenían de la deforestación ${ }^{6}$, especialmente de la Amazonia, pero también de otros ecosistemas, como el Cerrado brasileño. Lo anterior se debe a la matriz energética del país, caracterizada por ser de baja intensidad en carbono, en función de la mayor utilización de hidroeléctricas en la generación de electricidad y la progresiva sustitución del uso de la benzina y diesel por el etanol y biocombustibles (Viola, 2010).

Pese a los demás problemas en el ámbito ambiental, el gobierno de Lula da Silva logró reducir la deforestación de la Amazonia, especialmente en el periodo de 2005 hasta 2009, disminuyendo $25 \%$ de las emisiones de GEE, logrando la reducción en medio al contexto de un mayor crecimiento económico del país. Lo anterior fue posible debido a factores como: el aumento de la capacidad institucional del Estado en monitorear, fiscalizar y reprimir las actividades de deforestación; la creación de aéreas protegidas; la actuación de las ONG en concientizar a la opinión pública sobre los problemas generados por la deforestación, su influencia en la disminución del consumo de productos como soya y carne bovina provenientes de la región, incluso en el mercado internacional; y la mayor cooperación entre los gobiernos de las provincias amazónicas, junto al gobierno central. De este modo, entre los años 2005 y 2009, el país logró cambiar radicalmente su perfil y trayectoria de las emisiones, teniendo en cuenta su singular proceso al disminuirla gracias a la reducción de la deforestación en la región amazónica (Viola, 2010).

En el 2009, la Ley de Cambio Climático fue aprobada por el Congreso, lo cual internalizó parcialmente la problemática del cambio climático en el ordenamiento jurídico brasileño, aunque no se establecieron metas definitivas para la reducción de las emisiones de GEE. En el mismo año, se anunció la nueva posición del gobierno por medio de un compromiso voluntario de reducción, se comprometió a disminuir

5. La distribución de emisiones de GEE es fuertemente desequilibrada en las distintas regiones del país, ya que la región amazónica representa $40 \%$, pero concentra solamente $12 \%$ de la población, así como $7 \%$ del PIB nacional. Esto mientas que, en el resto del país, se concentran $60 \%$ de las emisiones, $88 \%$ de la población y $93 \%$ de PIB nacional, lo cual torna la asimetría en la distribución regional de las emisiones de Brasil, una de las mayores del mundo, en el mismo tiempo en que las emisiones a nivel per capita y la intensidad del uso de carbono en la región están entre los niveles más altos del planeta.

6. Cerca de $18 \%$ de las emisiones a nivel global provienen de la deforestación y del uso de la tierra; en este sentido, la deforestación en la Amazonia, que tiene gran importancia en el clico global del carbono, transforma Brasil en el segundo mayor emisor por desforestación y uso del suelo, responsable por $2 \%$ de las emisiones globales con este origen, solamente atrás de Indonesia, responsable por $4 \%$ de las emisiones (Viola, 2010). 
entre $36 \%$ y $39 \%$ las emisiones de acuerdo con las expectativas proyectadas para el 2020, lo cual representó la reducción de $20 \%$ con respecto a las emisiones del 2005 y $10 \%$ en relación con las del 2008, estimulados por la reducción de la deforestación en la Amazonia. Esto es importante, en la medida en que el país se colocaba en el selecto grupo de países que internacionalizaban la cuestión climática en su arquitectura jurídica, a semejanza de países como los miembros de la Unión Europea, Suiza, Noruega, Japón y Corea del Sur. No obstante, es importante resaltar que se trata de un compromiso voluntario, sin la disposición de asumirlo en el ámbito de un acuerdo internacional vinculante. Tampoco es una meta de reducción obligatoria con base en los años noventa, conforme los compromisos firmados por los países citados (Viola y Franchini, 2013).

\section{- La diversificación de la matriz energética}

Otra política importante en el ámbito de mitigación del cambio climático impulsada por el propio mandatario fue la utilización del etanol y otros biocombustibles en la transformación hacia una economía baja en carbono. Entretanto, el principal desafío fue garantizar que la transición hacia el uso de los biocombustibles no ampliara la deforestación ni disminuira la seguridad alimentaria del país. Esto se generó en el marco de las presiones internacionales, ya que la plantación de soya, materia prima fundamental para la producción del biodiesel, ha avanzado hacia la región amazónica. Además, para la comunidad internacional, el aumento de la producción del etanol en la región central de Brasil también estimularía la ampliación de la deforestación en la Amazonia, al desplazar la frontera agropecuaria a esta región (Viola, 2010).

Esta política pública lanzada en la década de 1970 y renovada por Lula da Silva, presentó un importante impacto en la trayectoria de las emisiones brasileñas y llegó a transformarse en una herramienta competitiva en el combate al cambio climático global. Así, en 2010, el etanol ya era responsable por cerca de $20 \%$ de la matriz energética brasileña y puso a Brasil atrás solamente del sector petrolero en el ámbito de los transportes.

De hecho, desde el 2006, Lula da Silva pasó a defender la constitución de una economía global de biocombustibles, donde destacó el posicionamiento del país en los debates internacionales sobre el cambio climático; sin embargo, perdió fuerzas con la descubierta de las reservas marítimas de petróleo en la camada del pre-sal a partir del 2008 (Viola y Franchini, 2013). 


\section{- La transición en el perfil de emisiones del país}

Desde el 2010, las emisiones volvieron a crecer debido a la fuerte expansión de diversos sectores de la economía y el mayor consumo de productos derivados del petróleo, como la bencina y el aumento de las actividades económicas e industriales que culminaron con el crecimiento de 7,5\% del PIB. De este modo, el perfil de emisiones brasileño cambió y se tornó similar al de otros países de renta mediana, a partir del mayor aumento de la participación relativa de los sectores más modernos de la economía, como energía, industria, agropecuaria y residuos en las emisiones de GEE. Por ende, este período inaugura una nueva etapa en el perfil de emisiones, pese la manutención de la matriz energética limpia del país (Viola y Franchini, 2013).

Asimismo, es importante señalar que la reducción de la desforestación como la principal fuente emisora de GEE genera nuevos retos para la transición hacia una economía baja en carbono y obliga a la nación a adoptar nuevas políticas de mitigación. Lo anterior ocurre debido a que, para lograr el descenso de las emisiones, Brasil tuvo que implementar políticas de control solo en la actividad ilícita que es la deforestación y no en su matriz productiva. Así, pasa a ser necesaria la introducción de otras opciones de mitigación, que requieren más recursos, inversiones, tecnologías, es decir, más caras y más semejantes a las economías más avanzadas, lo que amplía los costos en la transición hacia la economía baja en carbono (Viola y Franchini, 2013).

\section{- El regreso de las políticas conservadoras}

La trayectoria positiva generada por la internalización del Régimen del Cambio Climático en el ordenamiento interno brasileño pasó a desacelerarse a partir de 2011, en virtud de distintos factores como: la expansión del sector petrolero en el país, la adopción del nuevo Código Forestal brasileño ${ }^{7}$, la nueva política industrial de promoción a la industria automotriz ${ }^{8}$, la reducción en las tarifas de energía erétrica y los tributos de la bencina para evitar el alza del producto y estimular su

7. La reforma del Código Forestal fue en camino opuesto a las políticas de mitigación del cambio climático, ya que flexibilizó el marco regulatorio de los bosques, perdonó parcialmente a los grupos responsables por deforestación ilegal hasta 2008 y dejó evidente el poder de presión de grupos rurales conservadores (Viola y Franchini, 2013).

8. Además, las medidas de estímulo al crecimiento industrial implementadas a través de la reducción de tributos sobre los productos industrializados introducidas en 2012 no tomaron en cuenta ninguna condicionante de carácter climático o ambiental a ser exigida por parte de las industrias. De este modo, la prioridad del gobierno de Dilma Roussef, sucesora de Lula da Silva, estaba centrada en el crecimiento económico, con pocas referencias a la cuestión ambiental, agravadas por la desaceleración del crecimiento del país a partir de 2013 (Viola y Franchini, 2013). 
uso, la falta de avance en los proyectos establecidos por la Política Nacional sobre el Cambio Climático, entre otros. De este modo, la posición de carácter más reformista implementada a partir del 2009 retrocedía a posiciones más conservadoras en en 2011. Así, la política interna brasileña se ha tornado cada vez más divergente con los presupuestos de la agenda ambiental de los años anteriores, llegando a la indiferencia e incluso omisión del país ante una de serie de acciones. Por lo tanto, la cuestión climática pasaba a adquirir una posición marginal en la política interna brasileña (Viola y Franchini, 2013).

Igualmente, la construcción de hidroeléctricas en la región amazónica también originó problemas con organizaciones no gubernamentales y ambientalistas, puesto que el gobierno tampoco consideró los altos costos y los grandes impactos ambientales y sociales involucrados en las obras de explotación del potencial generador de energía hidroeléctrica en los ríos amazónicos ${ }^{9}$. Esto evidenciaba cada vez más la debilidad del Ministerio del Medioambiente frente a otros organismos estatales, al que le correspondía intentar mitigar los daños causados por la implementación de los proyectos. Además, la retomada del proyecto nuclear generó problemas junto al Ministerio del Medioambiente y su ministra de este entonces. Sobre el argumento de la necesidad de diversificar la matriz energética brasileña, utilizando fuentes no emisoras de GEE, fue planificada la construcción de otras centrales nucleares, que complementaban las ya existentes en el país, entretanto, sin el debate público respecto de sus construcciones. Apoyadas por el Ministerio de Ciencia y Tecnología, se evidenciaban las contradicciones entre las distintas carpetas ministeriales acerca de la preservación ambiental (Lisboa, 2011).

\section{Consideraciones finales}

Brasil ha pasado de una postura reactiva a una política exterior más activa, participativa y colaborativa, por lo cual ha logrado convertirse en uno de los protagonistas en la elaboración de los documentos en las grandes conferencias

9. En este campo, se centra la grande polémica relacionada con la construcción de la hidroeléctrica de Belo Monte, la cual repercutió incluso internacionalmente, en virtud los problemas técnicos y de los altos costos ambientales derivados de su construcción. El proyecto enfrentó la oposición de movimientos sociales organizados, logró movilizar los medios de comunicaciones nacionales e internacionales. Para muchos, el permiso de licenciamiento fue otorgado a través de presiones ilegitimas y sin la participación popular, como prevé la Comisión Mundial de Represas, atendiendo los intereses de las grandes empresas del sector de la construcción civil brasileña, bien como de los sectores que necesitan de gran cantidad de energía, como el minero y el metalúrgico, por ejemplo (Lisboa, 2011). 
multilaterales, especialmente en el régimen del cambio climático, teniendo siempre en cuenta los principios de las responsabilidades comunes, pero diferenciadas, y la preservación de la soberanía sobre sus recursos naturales. De este modo, para el país es importante la idea de preservar y proteger el medioambiente, pero considera que los países desarrollados deben financiar y equilibrar las relaciones de poder con los países en vías de desarrollo para no estancar las posibilidades de crecimiento de las naciones menos avanzadas.

Así, el país ha ejercido el liderazgo junto al Grupo de los 77, en la búsqueda de formalizar su posición de la política exterior en el marco de un grupo más amplio, manteniendo su liderazgo y postura. De este modo, pese a las contradicciones existentes entre sus políticas internas, el país logró un mayor protagonismo en las relaciones internacionales contemporáneas de principios del siglo XXI, sobre todo con la llegada de Lula da Silva a la presidencia, quien evidenció la importancia del país en la elaboración de la reglas multilaterales que influyen en la gobernanza global que beneficien tanto a los países desarrollados, como a las naciones en vías de desarrollo, así como la preservación del medioambiente, especialmente en el régimen del cambio climático.

\section{Referencias}

Amorin, C. (2010). Brazilian Foreign Policy under President Lula (2003-2010): an overview. Revista Brasileira de Política Internacional, 53, 214-240. doi: 10.1590/S0034-73292010000300013

Barros-Platiau, Ana Flávia. (2010). When emergent countries reform global governance of climate change: Brazil under Lula. Revista Brasileira de Politica Internacional, 53(spe), 73-90. doi: 10.1590/S0034-73292010000300005

Brito, R. S. (2011). A participação do Brasil no cenário das mudanças ambientais globais em Quioto, Copenhague e Cancún. Recuperado de: http:// cmb.adv.br/assets/a-participa\%C3\%A7\%C3\%A3o-do-brasil-no-cen\%$\mathrm{C} 3 \% \mathrm{~A} 1$ rio-das-mudan\%C3\%A7 as-ambientais-globais-em-quioto-copenhague-e-canc\% $\% 3 \% \mathrm{BAn}$ rafaela-silva-brito.pdf

Estenssoro, F. (2010). Crisis ambiental y cambio climático en la política global: Un tema crecientemente complejo para América Latina. Revista Universum, 25(2), 57-77. Recuperado de: http://www.scielo.cl/ 
scielo.php?script=sci_arttext\&pid=S0718-23762010000200005 doi: $10.4067 /$ S0718-23762010000200005

Estenssoro S., F. (2014). Historia del debate ambiental en la política mundial. 1945-1992. La perspectiva latinoamericana. Santiago: IDEA.

Kageyama, P, \& Santos, J. D. dos. (2012). Aspectos da política ambiental nos governos Lula. Revista FAAC - UNESP Bauru, 1(2).

Lisboa, M. V. (2011). Balanço da política ambiental do Governo Lula: Grandes e duradouros impactos. In M. de Paula (Org.), "Nunca antes na história desse pais"...?: um balanço das políticas do governo Lula. Rio de Janeiro, RJ: Heinrich Böll Stiftung.

Quirola, D. (2009). América Latina, el cambio climático y la ruta hacia Copenhague. Friedrich Ebert Stiftung: Proyecto Regional de Energía y Clima. Recuperado de: http://library.fes.de/pdf-files/bueros/quito/06814.pdf

Reggiani, M. (2008). O posicionamento político do Brasil no Regime de Mudanças Climáticas: Uma discussão de dois níveis. Pontifícia Universidade Católica de Minas Gerais, Fundação de Amparo à Pesquisa do Estado de Minas Gerais. Recuperado de: http://gedi.objectis.net/eventos-1/ilsabrasil2008/artigos/deri/reggiani.pdf

Viola, E. (2010). A política climática global e o Brasil: 2005-2010. Instituto de Pesquisa Econômica Aplicada (Ipea). Revista Tempo do Mundo (RTM): 2(2), 81-117. Recuperado de: http://ipea.gov.br/agencia/images/stories/PDFs/ rtm/110321_rtm_vol02_cap4.pdf

Viola, E. y Franchini, M. (2013). Brasil na Governança Global do Clima (2005-2012): A luta entre conservadores e reformistas. Revista Contexto Internacional, 35(1), 43-76. Recuperado de: http://www.scielo.br/scielo.php?script=sci_arttext\&pid=S0102-85292013000100002 doi: 10.1590/ S0102-85292013000100002

Visenti, P. G., Fagundes, S., Reis, A. (2010). Brazil and the Economic, Political, and EnvironmentalMultilateralism:theLulayears(2003-2010). RevistaBrasileirade Política Internacional,53(número especial),54-72. Recuperadode: http://www. scielo.br/scielo.php?script=sci_arttext\&pid=S0034-73292010000300004 